\title{
Fractional spectral integral methods for valuing cryptocurrency asset flow fractional differential equations.
}

\author{
Claude Moutsinga ${ }^{1}$,Edson Pindza ${ }^{1}$, and Eben Mare $^{1}$ \\ ${ }^{1}$ University of Pretoria
}

May 10, 2021

\begin{abstract}
Since its inception in 2009, the cryptocurrency market has grown considerably. Several authors have proposed models to explain the price movements of assets in this new emerging market. However, only few researches have been done using the dynamical approach. This paper proposes a robust time fractional spectral method for studying a three dimensional fractional differential equation that models cryptocurrency asset flow obtained by utilizing the concept of liquidity price. The method relies on fractional spectral integration matrix operator approach. Numerical simulations are conducted to show efficiency of the numerical method on the fractional cryptocurrency model compared to existing methods.
\end{abstract}

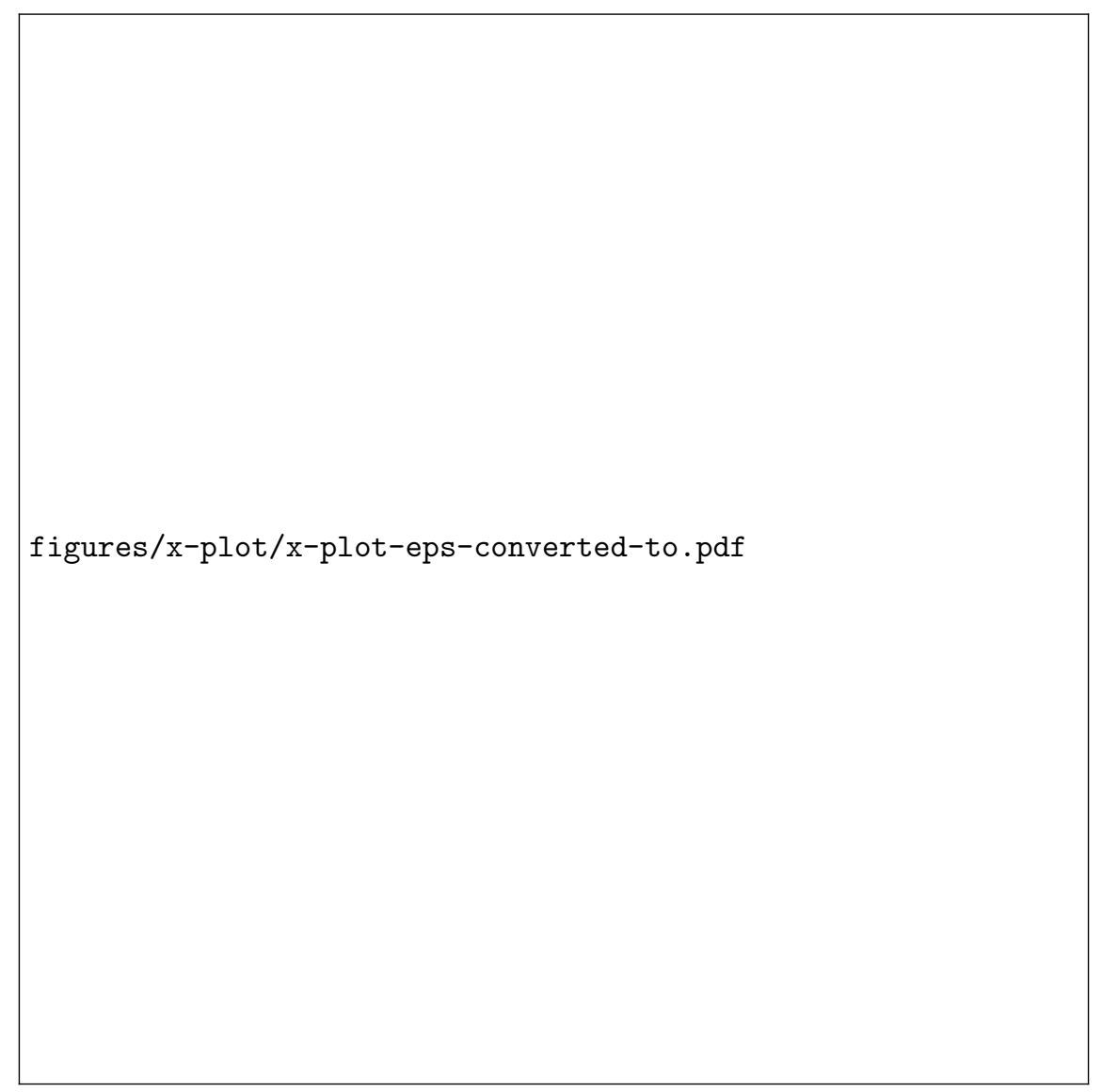


figures/y-plot/y-plot-eps-converted-to.pdf 
figures/z-plot/z-plot-eps-converted-to.pdf 
figures/errorz-plot/errorz-plot-eps-converted-to.pdf 
figures/x-plot1/x-plot1-eps-converted-to.pdf 
figures/y-plot2/y-plot2-eps-converted-to.pdf 
figures/z-plot1/z-plot1-eps-converted-to.pdf 
figures/xyz-plot/xyz-plot-eps-converted-to.pdf 
figures/xy-phase/xy-phase-eps-converted-to.pdf 
figures/xz-phase/xz-phase-eps-converted-to.pdf 
figures/yz-phase/yz-phase-eps-converted-to.pdf 
figures/x-plot-alphas/x-plot-alphas-eps-converted-to.pdf 
figures/y-plot-alphas/y-plot-alphas-eps-converted-to.pdf 
figures/z-plot-alphas/z-plot-alphas-eps-converted-to.pdf 
figures/efficiencyZ/efficiencyZ-eps-converted-to.pdf 
figures/errorz-plot2/errorz-plot2-eps-converted-to.pdf 